\title{
Joint Replenishment Problem in Multi-ltem Inventory Control with Carrier Capacity and Receiving Inspection Cost
}

\author{
Keisuke Nagasawa \\ Department of Information and Communication Sciences, Sophia University, Tokyo, Japan \\ Email: nagasa-k@sophia.ac.jp \\ Takashi Irohara \\ Department of Information and Communication Sciences, Sophia University, Tokyo, Japan \\ Email: irohara@ sophia.ac.jp (Corresponding Author)
}

\author{
Yosuke Matoba, Shuling Liu
}

Fairway Solutions Inc

\begin{abstract}
In the present paper, a multi-item inventory problem with the capacity to replenish carriers is considered. In the present study, we propose a model that considers truck capacity, truck cost, and receiving inspection cost with inventories and shortages in inventory management simultaneously. A number of studies have examined the minimum total cost while considering the ordering cost as fixed and not considering the carrier capacity or receiving inspection cost. However, we consider the stepwise ordering cost, which changes in a stepwise manner according to the ordered quantity and the capacity of the carrier. In addition, we consider a receiving inspection cost that is defined not only by ordered quantity but also by ordered item variety. The problem considered herein was formulated as a mathematical programming problem in a finite planning period, and valid inequalities were considered. We could consider individual objective functions for ordering, holding, lost sales, and receiving inspection cost together. We can observe that the receiving inspection cost could be reduced by aggregating the order quantity.
\end{abstract}

Keywords: inventory management, joint replenishment problem, mathematical programming, valid inequality

\section{INTRODUCTION}

Effective inventory management has played an important role in the success of supply chain management. As such, inventory management has been investigated by numerous researchers and practitioners. Moreover, ordering policies, the method for setting parameters, and the method of demand forecasting, for example, have also been investigated extensively.

In inventory management, deciding the timing of ordering and the amounts of items to be ordered (i.e., deciding the ordering policy) is important. Several studies have analyzed the case of a single item and methods for determining the ordering policy and parameters under stable stochastic distribution demand (e.g., the reorder point ordering policy under Bernoulli demand with random lead time by Shinha (2012) and the base-stock ordering policy for multi-echelon inventory management under Poisson demand with an Erlang distribution replenishment process by Ntio and Vidalis (2011)). However, if a single ordering policy is applied to several different items that have different shipping statistics, then the inventory will be a mix of items with shortages and excess inventory.

Generally, proper ordering policies for each item are considered to result in a reduction in inventory and a reduction in shortages of items. However, when the inventory manager orders numerous items from the same company, the ordering cost charged depends on the number of ordering item variety and ordering frequency. Therefore, the order quantity of each item cannot be decided freely. This problem is referred to as the joint replenishment problem (JRP). The typical assumptions of the JRP are similar to the economic ordering quantity. The demand is deterministic and constant, and shortages are not allowed. There are no quantity discounts, and the holding cost is linear. Goyal (1974) developed an algorithm to determine the optimal solution under most classical assumptions. Then, Silver (1976) developed an efficient heuristic algorithm for solving the JRP, and Kaspi and Rosenblatt (1991) later improved the heuristic algorithm. Reviews of the literature on the JRP were conducted by Goyal and Satir (1989) and Khouja and Goyal (2008).

Another variety of inventory management is the full truckload problem. Trucks with a finite capacity are used to deliver products. The cost of ordering and transportation changes stepwise according to the ordered quantity and the capacity of the carrier. Kiesmüller (2009) proposed a dynamic order-up-to policy that provides full truckloads. Kiesmüller (2010) provided approximation formulas for computing the parameters of the replenishment policy, which takes into 
account the capacity restrictions of the total order volume, such that the given target service levels can be met.

A well-known inventory problem is the single warehouse multi-retailer (SWMR) problem as Chan et al. (2002). In the general SWMR problem, retailers know the external demand of products over a finite planning horizon. Items are shipped from the suppliers to the warehouse and are distributed from the warehouse to the retailers. The goal of solving this problem is to find an optimal replenishment timing and quantity to minimize the total transportation cost or inventory costs in the system. Yang et al. (2012) applied a genetic algorithm to the problem, which involves a known multiple-item demand, multiple retailers, a finite planning horizon, cost functions having discounts, and an optimized total cost.

Moreover, when inventory manager receives ordered items from supplier, inventory manager should inspect receiving items. The receiving inspection cost for each item is not always linearly proportional to the quantity ordered, but rather may be proportional to the variety of items. In such a situation, if the inventory manager frequently orders small quantities of numerous items, the receiving inspection cost might be high. In contrast, if the inventory manager orders a large quantity at one time in order to reduce the receiving inspection cost, items will become overstocked. Moreover, the cost and inventory movement also change based on the timing and quantity of items ordered.

In the present study, we formulate a joint replenishment problem as a mathematical programming problem within a finite planning horizon with carrier capacity, shortage cost due to lost sales, and receiving inspection cost. The types of orders that can reduce the shipping, inventory, shortage, and receiving inspection cost are clarified. Moreover, we apply valid inequalities to the formulation and compared the obtained results to the results without valid inequalities.

The remainder of the present paper is organized as follows. In Section 2, the mathematical formulation and valid inequalities of the model are presented, and the computational results are reported in Section 3. Finally, conclusions are presented in Section 4.

\section{MODEL DESCRIPTION}

The features of the present study are described below, and we formulate the problem as follows. We consider a multi-item inventory management problem in which a warehouse sells multiple items with demand and periodic replenishment from a supplier during a finite planning period. For each order, we use a carrier with a fixed capacity, such as a truck. A number of studies have considered the ordering cost to be a fixed cost. However, we herein consider a stepwise ordering cost, which changes stepwise according to the ordered quantity and the capacity of the carrier. Thus, the ordering cost is calculated as the cost of the truck multiplied by the number of trucks used. Quantity discounting is not carried out. Thus, a full load is not necessary. Items can be stored until the next period and do not deteriorate. Moreover, shortage can be applied to some orders. Usually, shortage can be categorized as either backorders or lost sales. Backorders occur when a shortage occurs and the customer must wait until the next arrival of items, and lost sales occur when a shortage occurs when a customer cancels an order immediately if the items are not available. In the present study, we consider lost sales. Moreover, we also consider the receiving inspection cost. Upon receiving items, a cost for receiving inspection is incurred. This receiving inspection cost is independent of the amount of the order but is dependent on the variety of items ordered. The ordering, inventory, receiving inspection, and shortage costs are considered as objective functions in the present study.

\subsection{Model formulation}

(1) Decision variables

$y_{t}$ number of carriers used on day $t$

$s_{t}^{i} \quad$ quantity of lost sales of item $i$ on day $t$

$l_{t}^{i} \quad$ inventory level of item $i$ on day $t$

$x_{t}^{i} \quad$ quantity of item $i$ ordered on day $t$

$r_{t}^{i} \quad$ whether item $i$ is received on day $t$

$z_{t}^{i} \quad$ whether item $i$ is received or a shortage has occurred on day $t$

\section{(2) Parameters}

$\begin{array}{ll}u_{t} & \text { usage fee of the carrier on day } t \\ p_{t}^{i} & \text { penalty for lost sales of item } i \text { on day } t \\ h_{t}^{i} & \text { holding cost of one unit item } i \text { on day } t \\ a_{t}^{i} & \text { receiving inspection cost of one unit item } i \text { on day } t \\ d_{t}^{i} & \text { demand of item } i \text { on the day } t \\ v^{i} & \text { volume of one unit for item } i \\ c & \text { capacity of the carrier } \\ I & \text { set of items } \\ T & \text { set of days }\end{array}$

\section{(3) Objective functions and constraint}

In the present study, we could formulate, simply, the multi-item inventory problem as follows:

$$
\begin{array}{ll}
\text { minimize } & f_{1}=\sum_{\forall t \in T} u_{t} y_{t} \\
\text { minimize } & f_{2}=\sum_{\forall i \in I} \sum_{\forall t \in T} h_{t}^{i} l_{t}^{i} \\
\text { minimize } & f_{3}=\sum_{\forall i \in I} \sum_{\forall t \in T} p_{t}^{i} s_{t}^{i} \\
\text { minimize } & f_{4}=\sum_{\forall i \in I} \sum_{\forall t \in T} a_{t}^{i} r_{t}^{i}
\end{array}
$$


subject to

$$
\begin{array}{ll}
l_{t-1}^{i}+x_{t}^{i}-l_{t}^{i}+s_{t}^{i}=d_{t}^{i} & \forall i \in I, \forall t \in T \\
\sum_{\forall i \in I} v^{i} x_{t}^{i} \leq c y_{t} & \forall t \in T \\
s_{t}^{i} \leq d_{t}^{i} & \forall i \in I, \forall t \in T \\
x_{t}^{i} \leq \mathrm{M} r_{t}^{i} & \forall i \in I, \forall t \in T \\
y_{t}, s_{t}^{i}, l_{t}^{i}, x_{t}^{i} \in \mathrm{Z}_{+} & \forall i \in I, \forall t \in T \\
r_{t}^{i} \in\{0,1\} & \forall i \in I, \forall t \in T
\end{array}
$$

There are four objective functions, which are given by Eqs. (1) through (4). For each objective function, we consider the sum of each item and each planning period. The first objective function, Eq. (1), considers the transportation cost and usage fee of the truck, and the second objective function, Eq. (2), considers the holding cost. The third objective function, Eq. (3), considers the shortage cost, and the fourth objective function, Eq. (4), considers the receiving inspection cost. Equation (5) is the inventory balancing equation for all items. Equation (6) is the constraint on the order quantity and the volume of the truck that can be loaded for all items within a planning period. During each period, the volume of the sum of ordered items cannot exceed a multiple of the volume of the truck and the number of trucks. Equation (7) is the constraint on excessive shortage. Equation (8) is the constraint checking ordering or not. The constraint is necessary for calculating receiving inspection for all items within each planning period.

\section{(4) Solution method and valid inequalities}

If the weight of each of the objective functions is defined, the objective functions can be combined in a linear combination, and a single objective function is obtained. If the objective function is a single linear function, we can solve the problem using a mathematical programming solver, for example. We optimized the objective function given by Eq. (9), which is a weighted linear combination of the four objective functions weighted by $\alpha, \beta, \omega$, and $\gamma$.

In the constraint given by Eq. (8), $\mathrm{M}$ is a sufficiently large number. Since, in this problem, we can use the total demand instead of $\mathrm{M}$, we use the following equation instead of Eq. (8):

$$
x_{t}^{i} \leq\left(\sum_{\forall t \in T} d_{t}^{i}\right) r_{t}^{i} \quad \forall i \in I, \forall t \in T
$$

$$
\underset{\forall t \in T}{\operatorname{minimize}} \quad \alpha \sum_{t} u_{t}+\beta \sum_{\forall i \in I} \sum_{\forall t \in T} h_{t}^{i} l_{t}^{i}+\omega \sum_{\forall i \in I} \sum_{\forall t \in T} p_{t}^{i} s_{t}^{i}+\gamma \sum_{\forall i \in I} \sum_{\forall t \in T} a_{t}^{i} r_{t}^{i}
$$

Although the problem can be solved using the above formula, in order to improve the accuracy of solution, we formulate the following valid inequalities:

$$
\begin{array}{ll}
\sum_{\forall i \in I} v^{i} x_{t}^{i} \geq c\left(y_{t}-1\right) & \forall t \in T \\
y_{t} \geq r_{t}^{i} & \forall i \in I, \forall t \in T \\
\sum_{\forall t \in T} x_{t}^{i}+\sum_{\forall t \in T} s_{t}^{i}=\sum_{\forall t \in T} d_{t}^{i} & \forall i \in I, \\
x_{t}^{i}+s_{t}^{i} \leq\left(\sum_{\forall t \in T} d_{t}^{i}\right) z_{t}^{i} & \forall i \in I, \forall t \in T \\
x_{t}^{i}+s_{t}^{i} \leq d_{t}^{i} z_{t}^{i}+l_{t}^{i} & \forall i \in I, \forall t \in T \\
d_{t}^{i}-d_{t}^{i} z_{t}^{i} \leq l_{t-1}^{i} & \forall i \in I, \forall t \in T \\
z_{t}^{i} \in\{0,1\} & \forall i \in I, \forall t \in T
\end{array}
$$

Equation (10) is the constraint on using the minimum number of trucks corresponding to the volume of items ordered. In Eq. (11), if an order is received in a period, then a truck is used. In Eq. (12), the sum of ordered items and the sum of the shortage are equal to the sum of demand. Eq. (13) can be used to determine whether an order has been placed or a shortage has occurred. Using Eq. (13), the constraints given by Eqs. (14) and (15) can be used to restrict the inventory level of each item during each period. Using Eq. (14), when no order has been placed and no shortage has occurred, $z_{t}^{i}=0$, the inventory is greater than 0 . On the other hand, when an order has been placed or a shortage has occurred, $z_{t}^{i}=1$, the sum of the demands and inventories becomes greater than the sum of the order quantity and quantity of the shortage of items of each item during every period. Using Eq. (15), when no order has been placed and no shortage has occurred, $z_{t}^{i}=0$, the inventories from the last period to the current period are greater than the demand quantity of the current period. On the other hand, when an order is placed or a shortage has occurred, $z_{t}^{i}=$ 1 , the inventory from the last period to the current period is greater than 0 .

\subsection{Data set}

We run the Gurobi Optimizer 5.5.0 solver on an Intel® Core $^{\mathrm{TM}}$ i7-3370 CPU at $3.40 \mathrm{GHz}$ (Memory: $16 \mathrm{~GB}$ ) to solve the mixed integer programming model. We used 
constant-demand data for seven items. The demands were $10,30,50,70,90,110$, and 130 . We set the truck capacity to 300 and ran the experiment for 50 iterations. We varied the weights $\alpha, \beta, \omega$, and $\gamma$, for the individual functions of interest in order to obtain several Pareto solutions. We calculated each case until the gap became less than $0.5 \%$ or until the computation time exceeds 600 seconds.

\section{NUMERICAL EXPERIMENTS}

Table 1 shows the weights, results, computation times, and gaps (as calculated by [upper bound - lower bound] / upper bound) of each objective function. For the computation time and gap, we compared the formulations of the present study with and without valid

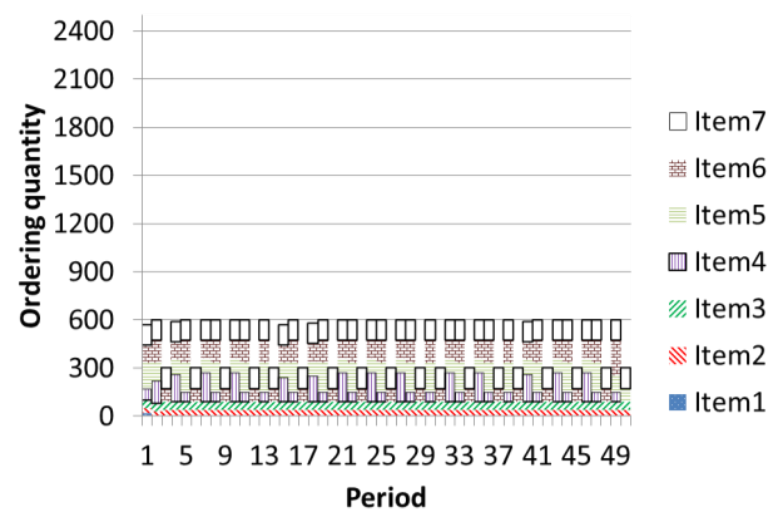

Figure 1. Ordered quantity of each item in case $12(\omega=0.00)$

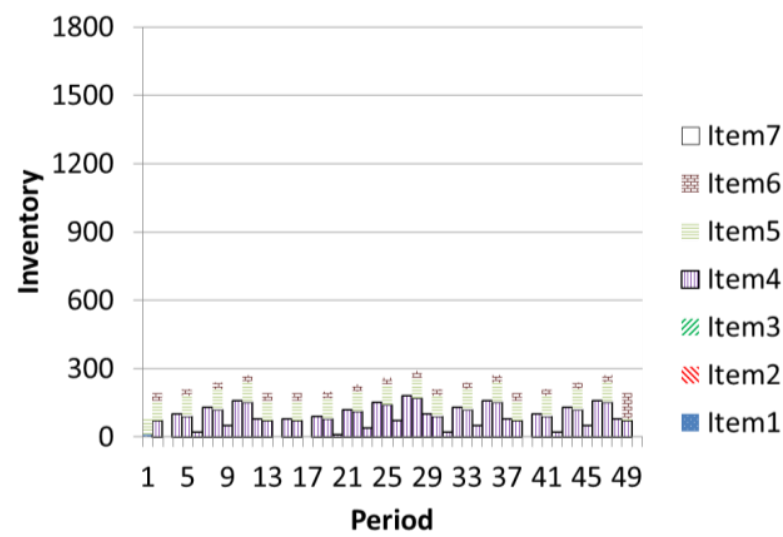

Figure 3. Inventory of each item in case $12(\omega=0.00)$

We next discuss the solutions obtained for different weights of the receiving inspection cost for case $12(\omega=$ $0.00)$ and case $9(\omega=0.80)$. The ordered quantities for case 12 are shown in Figure 1, and the inventories for case $12(\omega=0.00)$ are shown in Figure 3. If the truck and holding costs are weighted and the receiving inspection cost is ignored (case 12), then an appropriate policy is to order volume will equal truckload of one or two truck inequalities.

Table 1 revealed that if the weight of the shortage, $\gamma$, is reduced, the number of trucks, the inventory, and the receiving inspection cost become minimum (cases 1 and 2 ). This is because if the shortage disregarded, we can reduce the number of trucks, the inventory, and the receiving inspection cost by incurring a low shortage cost.

As shown in Table 1, except for cases 10 and 25, the valid inequalities improve the gap. Because the weight of the inventory is relatively high in cases 10 and 25 , we could assume that the valid inequalities of Eqs. (14) and (15) could not contribute to improve solution in the case where trucks and receiving inspection costs are balanced and considering the number of inventory.

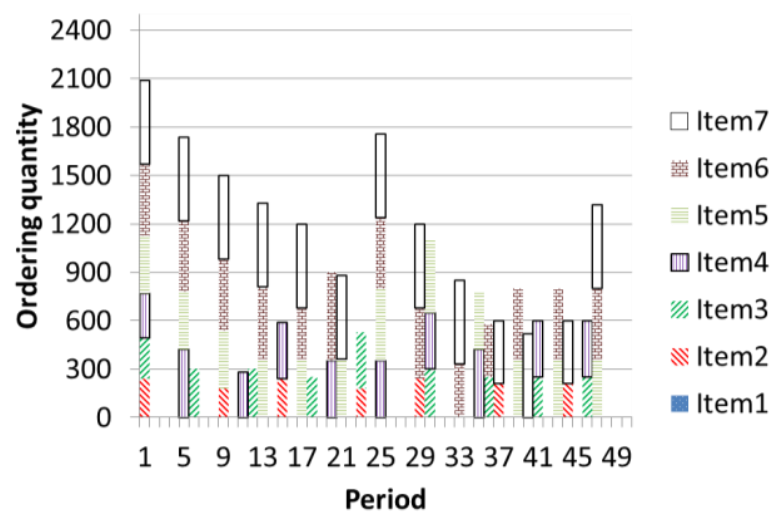

Figure 2. Ordered quantity of each item

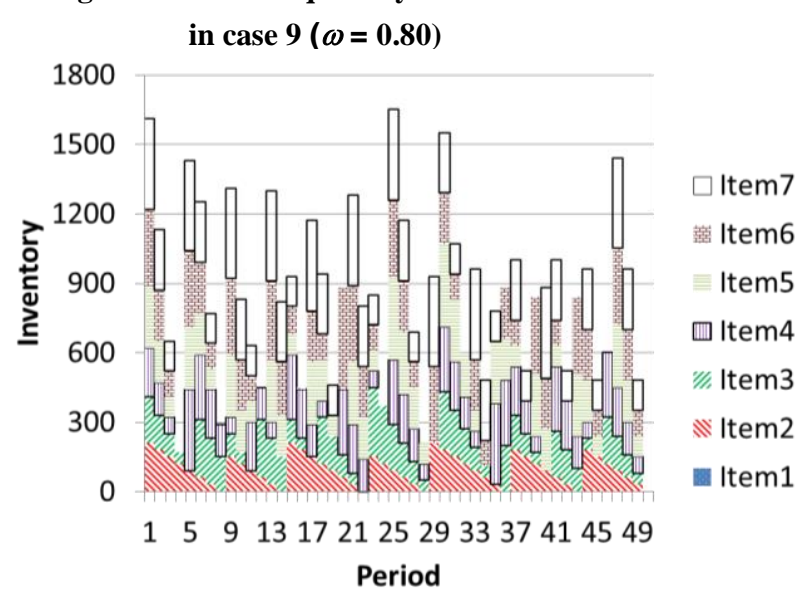

Figure 4. Inventory of each item in case $9(\omega=0.80)$

and reduce the shipping and holding costs. As shown in Figure 1, trucks (one or two trucks) are almost full load during each period.

Next, we show the ordered quantity and inventory for case $9(\omega=0.80)$, in which the receiving inspection cost is weighted. The ordered quantities for case 9 are shown in Figure 2, and the inventories for case 9 are shown in Figure 4. From comparison of Figure 3 and Figure 4, the 
quantity of inventory increases. Moreover, comparison of Figures 1 and 2 reveals that aggregating the number of ordered items can reduce the total receiving inspection cost of the ordered items. As a result, for different weights of the receiving inspection cost, as shown in
Figures 3 and 4, the sum of the inventories increases under receiving inspection cost. Therefore, when we consider the large receiving inspection cost, both the timing of the ordered quantity and the period of consuming the inventory of the order will change.

Table 1. Each weight of objective function, the result of each function, computation time and gap of objective function

\begin{tabular}{|c|c|c|c|c|c|c|c|c|c|c|c|c|}
\hline \multirow[b]{2}{*}{ Case } & \multicolumn{4}{|c|}{ Weight } & \multicolumn{4}{|c|}{ Objective function (with valid inequalities) } & \multicolumn{2}{|c|}{ Computation time $[\mathrm{s}]$} & \multicolumn{2}{|c|}{$\operatorname{Gap}(\%)$} \\
\hline & $\begin{array}{c}\text { Truck } \\
(\alpha)\end{array}$ & $\begin{array}{c}\text { inventory } \\
(\beta)\end{array}$ & $\begin{array}{c}\text { Receiving } \\
\& \text { inspection } \\
(\omega)\end{array}$ & $\begin{array}{c}\text { shortage } \\
(\gamma)\end{array}$ & $\begin{array}{l}\text { \# of } \\
\text { truck }\end{array}$ & $\begin{array}{c}\text { \# of } \\
\text { inventory }\end{array}$ & \begin{tabular}{|c|}
$\#$ of \\
receiving \\
inspection
\end{tabular} & $\begin{array}{c}\text { \# of } \\
\text { shortage }\end{array}$ & \begin{tabular}{c|} 
without \\
valid \\
inequalities \\
\end{tabular} & \begin{tabular}{c||} 
with \\
valid \\
inequalities
\end{tabular} & \begin{tabular}{c|} 
without \\
valid \\
inequalities \\
\end{tabular} & $\begin{array}{c}\text { with } \\
\text { valid } \\
\text { inequalities } \\
\end{array}$ \\
\hline 1 & $0.00 \sim 1.00$ & $0.0001 \sim 0.01$ & $0.00 \sim 1.00$ & 0.0001 & 0 & 0 & 0 & 24500 & 0.02 & 0.00 & $0.00 \%$ & $0.00 \%$ \\
\hline 2 & $0.00 \sim 1.00$ & $0.0001 \sim 0.01$ & $0.00 \sim 1.00$ & 0.001 & 0 & 0 & 0 & 24500 & 0.02 & 0.02 & $0.00 \%$ & $0.00 \%$ \\
\hline 3 & 0.00 & 0.0001 & 1.00 & 0.01 & 127 & 181690 & 20 & 0 & 600.00 & 600.00 & $9.14 \%$ & $1.13 \%$ \\
\hline 4 & 0.20 & 0.0001 & 0.80 & 0.01 & 83 & 164970 & 22 & 0 & 600.00 & 600.00 & $6.98 \%$ & $2.45 \%$ \\
\hline 5 & 0.50 & 0.0001 & 0.50 & 0.01 & 82 & 123760 & 29 & 0 & 600.00 & 600.00 & $2.31 \%$ & $0.72 \%$ \\
\hline 6 & 0.80 & 0.0001 & 0.20 & 0.01 & 82 & 79560 & 45 & 0 & 600.00 & 600.00 & $1.34 \%$ & $1.13 \%$ \\
\hline 7 & 1.00 & 0.0001 & 0.00 & 0.01 & \begin{tabular}{l|l|}
82 &
\end{tabular} & 6530 & 349 & 0 & 59.65 & 178.49 & $0.50 \%$ & $0.50 \%$ \\
\hline 8 & 0.00 & 0.001 & 1.00 & 0.01 & 111 & 46280 & 59 & 500 & 2.27 & 3.47 & $0.39 \%$ & $0.00 \%$ \\
\hline 9 & 0.20 & 0.001 & 0.80 & 0.01 & 84 & 42250 & 64 & 500 & 600.00 & 600.00 & $1.03 \%$ & $0.97 \%$ \\
\hline 10 & 0.50 & 0.001 & 0.50 & 0.01 & 81 & 29800 & 84 & 560 & 600.00 & 600.00 & $1.46 \%$ & $1.47 \%$ \\
\hline 11 & 0.80 & 0.001 & 0.20 & 0.01 & 82 & 17310 & 136 & 60 & 600.00 & 600.00 & $1.87 \%$ & $1.53 \%$ \\
\hline 12 & 1.00 & 0.001 & 0.00 & 0.01 & 83 & 4960 & 347 & 0 & 326.05 & 206.12 & $0.50 \%$ & $0.50 \%$ \\
\hline 13 & 0.00 & 0.01 & 1.00 & 0.01 & 50 & 0 & 100 & 12500 & 0.05 & 0.05 & $0.00 \%$ & $0.00 \%$ \\
\hline 14 & 0.20 & 0.01 & 0.80 & 0.01 & 50 & 0 & 100 & 12500 & 0.50 & 0.50 & $0.40 \%$ & $0.29 \%$ \\
\hline 15 & 0.50 & 0.01 & 0.50 & 0.01 & 100 & 0 & 200 & 4500 & 600.00 & 600.00 & $2.43 \%$ & $2.34 \%$ \\
\hline 16 & 0.80 & 0.01 & 0.20 & 0.01 & 100 & 0 & 300 & 500 & 0.05 & 0.05 & $0.00 \%$ & $0.00 \%$ \\
\hline 17 & 1.00 & 0.01 & 0.00 & 0.01 & 100 & 0 & 350 & 0 & 0.00 & 0.02 & $0.00 \%$ & $0.00 \%$ \\
\hline 18 & 0.00 & 0.1 & 1.00 & 0.01 & 50 & 0 & 100 & 12500 & 0.05 & 0.03 & $0.00 \%$ & $0.00 \%$ \\
\hline 19 & 0.20 & 0.1 & 0.80 & 0.01 & 50 & 0 & 101 & 12440 & 0.42 & 0.47 & $0.40 \%$ & $0.47 \%$ \\
\hline 20 & 0.50 & 0.1 & 0.50 & 0.01 & 100 & 0 & 200 & 4500 & 600.00 & 600.00 & $2.00 \%$ & $1.99 \%$ \\
\hline 21 & 0.80 & 0.1 & 0.20 & 0.01 & 100 & 0 & 300 & 500 & 0.05 & 0.05 & $0.00 \%$ & $0.00 \%$ \\
\hline 22 & 1.00 & 0.1 & 0.00 & 0.01 & 100 & 0 & 350 & 0 & 0.02 & 0.03 & $0.00 \%$ & $0.00 \%$ \\
\hline 23 & 0.00 & 0.001 & 1.00 & 0.1 & 113 & 49180 & 63 & 0 & 1.53 & 4.88 & $0.48 \%$ & $0.46 \%$ \\
\hline 24 & 0.20 & 0.001 & 0.80 & 0.1 & 87 & 44990 & 68 & 0 & 600.00 & 600.00 & $1.13 \%$ & $1.08 \%$ \\
\hline 25 & 0.50 & 0.001 & 0.50 & 0.1 & 83 & 32990 & 89 & 0 & 600.00 & 600.00 & $1.73 \%$ & $1.93 \%$ \\
\hline 26 & 0.80 & 0.001 & 0.20 & 0.1 & 82 & 17830 & 138 & 0 & 600.00 & 600.00 & $2.07 \%$ & $1.87 \%$ \\
\hline 27 & 1.00 & 0.001 & 0.00 & 0.1 & 83 & 4960 & 320 & 0 & 144.93 & 227.13 & $0.50 \%$ & $0.50 \%$ \\
\hline 28 & $0.00 \sim 1.00$ & 0.1 & $0.00 \sim 1.00$ & 0.1 & 100 & 0 & 350 & 0 & 0.03 & 0.05 & $0.00 \%$ & $0.00 \%$ \\
\hline
\end{tabular}

\section{CONCLUSION}

In the present paper, a multi-item inventory problem that considers the capacity of replenishing carriers is considered. In the present study, we proposed a model that considers truck capacity, truck cost, and receiving inspection cost with inventories and shortages in inventory management simultaneously. A number of studies have attempted to achieve minimum total cost while considering the ordering cost to be fixed and ignoring carrier capacity and receiving inspection cost. However, we herein considered a stepwise ordering cost that changes according to the ordered quantity and the carrier capacity. Moreover, we considered a receiving inspection cost that is defined in terms of both the quantity and variety of items ordered.

Using the proposed model, we could consider the individual objective functions for ordering, holding, lost sales, and receiving inspection costs, together in the deterministic demand joint replenishment problem.

Experimental results reveal that when the receiving inspection cost is considered, the order was aggregated, even if the ordered quantity and storage are increased.

In the future, since the structure of the receiving inspection cost will change according to the inspection procedure for each item and the relationship between items, modeling of the receiving inspection cost and the development of heuristic methods are also desired.

\section{REFERENCES}

Chan, L. M. A., Muriel, A., \& Shen, Z. J. M. (2002), Effective zero-inventory-ordering policies for the single-warehouse multi retailer problem with piecewise linear cost structures, Management Science, 48 (11), pp. 1446-1460. 
Goyal, S.K., (1974), Determination of optimum packaging frequency of items jointly replenished, Management Science, 21 (4), pp. 436-443.

Goyal, S.K., Satir, A.T., (1989), Joint replenishment inventory control: Deterministic and stochastic models, European Journal of Operational Research, 38 (1), pp. 2-13.

Kaspi, M., Rosenblatt, M.J., (1991), On the economic ordering quantity for jointly replenishment items, International Journal of Production Research, 29 (1), pp. 107-114.

Kiesmüller. G.P., (2009), A multi-item periodic replenishment policy with full truckloads, International Journal of Production Economics, 118 (1), pp. 275-281.

Kiesmüller. G.P., (2010), Multi-item inventory control with full truckloads: A comparison of aggregate and individual order triggering, European Journal of Operational Research, 200 (1), pp. 54-62.

Moutaz, K., and Goyal, S., (2008), A review of the joint replenishment problem literature: 1989-2005, European Journal of Operational Research, 186 (1), pp. 1-16.

Nito, D.D., and Vidalis M.J., (2011), Base-Stock Policies for Two and Three Stages Serial Inventory Systems with Stochastic Demand and Replenishments, Operations and Supply Chain Management, 4 (2), pp.78-84.

Silver, E.A., (1976), A simple method of determining order quantities in joint replenishments under deterministic demand, Management Science, 22 (12), pp. 1351-1361.

Sinha, P., (2012), A Note on Extension of a Bernoulli Demand Inventory Model, Operations and Supply Chain Management, 5 (1), pp. 54-58.

Yang, W., Chan, F.T.S., \& Kumar, V., (2012), Optimizing replenishment polices using Genetic Algorithm for singlewarehouse multi-retailer system, Expert Systems with Applications, 39 (3), pp. 3081-3086.

Keisuke Nagasawa a B.Eng. (2010) degree in the Department of Mechanical Engineering at Sophia University, Tokyo. He received a M.Eng. (2012) degree in the Department of Information and Communication Sciences in the Graduate School of Science and Technology of the same institution and is currently (2012-present) a Ph.D. candidate in the same department. His research interests include inventory management, supply chain management, optimization, and simulation. His research has appeared in Journal of Industrial Engineering \& Management Systems (IEMS) and Proceedings of Asia Pacific Industrial Engineering and Management Systems Conference (APIEMS), and at IIE Annual Conference and International Symposium on Reliability Engineering and Risk Management (ISRERM).

Takashi Irohara is a Professor at Sophia University in the Department of Information and Communication Sciences. He received his B. Eng., M. Eng., and Ph.D. in the Department of Industrial and Management Systems Engineering of Waseda University, Tokyo. His research interests include facility layout and material handling, manufacturing scheduling and logistics optimization. His papers have been published in International Journal of Biomedical Soft Computing and Human Sciences, Computers and Industrial Engineering, Journal of Industrial Engineering \& Management Systems (IEMS), Journal of Japan Industrial Management Associations, Transactions of the Japan Society of Mechanical Engineers and elsewhere. He received the Kimura Award from Transdisciplinary Federation of Science and Technology in 2013.

Yosuke Matoba is a software designer at Fairway Solutions Inc. He received his B.S. in Electric Engineering from the University of Tokyo.

Shuling Liu is a software designer at Fairway Solutions Inc. He received his M.S. in Mathematical Sciences from Kyoto University. 